\title{
Post-Release Capacitance Enhancement in Micromachined Devices
}

\author{
Cenk Acar \\ Microsystems Laboratory \\ University of California, Irvine \\ Irvine, CA, USA \\ cacar@uci.edu
}

\author{
Andrei M. Shkel \\ Microsystems Laboratory \\ University of California, Irvine \\ Irvine, CA, USA \\ ashkel@uci.edu
}

\begin{abstract}
This paper reports a novel design concept that aims to increase the detection and actuation capacitances in micromachined devices beyond the fabrication process limitations. The approach is based on designing the stationary components of the electrodes attached to a moving stage that permanently locks into the desired position before the operation of the device, to minimize the electrode gap with a simple assembly step. The concept has been implemented in bulk-micromachined prototype gyroscopes, and the experimental results have successfully demonstrated the feasibility of the assembly concept. It is experimentally shown that over an order of magnitude of capacitance increase is achieved in the same foot-print of the device, without additional fabrication steps.
\end{abstract}

\section{INTRODUCTION}

In the micro-domain, capacitive sensing and actuation offer several benefits when compared to other sensing and actuation means (piezoresistive, piezoelectric, optical, magnetic, etc.) with their ease of fabrication and integration, good DC response and noise performance, high sensitivity, low drift, and low temperature sensitivity.

The performance of micromachined sensors employing capacitive detection is generally determined by the nominal capacitance of the sensing electrodes. Even though increasing the overall sensing area provides improved sensing capacitance, the sensing electrode gap is the foremost factor that defines the upper bound. Similarly, the force generated by the electrostatic actuation electrodes (comb-drives or parallel-plates) is limited by the minimum gap attainable in the fabrication process. In electrostatically actuated devices such as micromachined gyroscopes, the nominal actuation capacitance determines the required drive voltages. For a small actuation capacitance, large voltages are needed to achieve sufficient forces, which in turn results in a large drive signal feed-through.

Various advanced fabrication technologies have been reported to minimize electrode gap, based on deposition of thin layers on electrode sidewalls [1, 2, 3]. For example, in one reported approach, high aspect-ratio polysilicon structures are created by refilling deep trenches with polysilicon deposited over a sacrificial oxide layer. Thick single-crystal silicon structures are released from the substrate through the front side of the wafer by means of a combined direc-
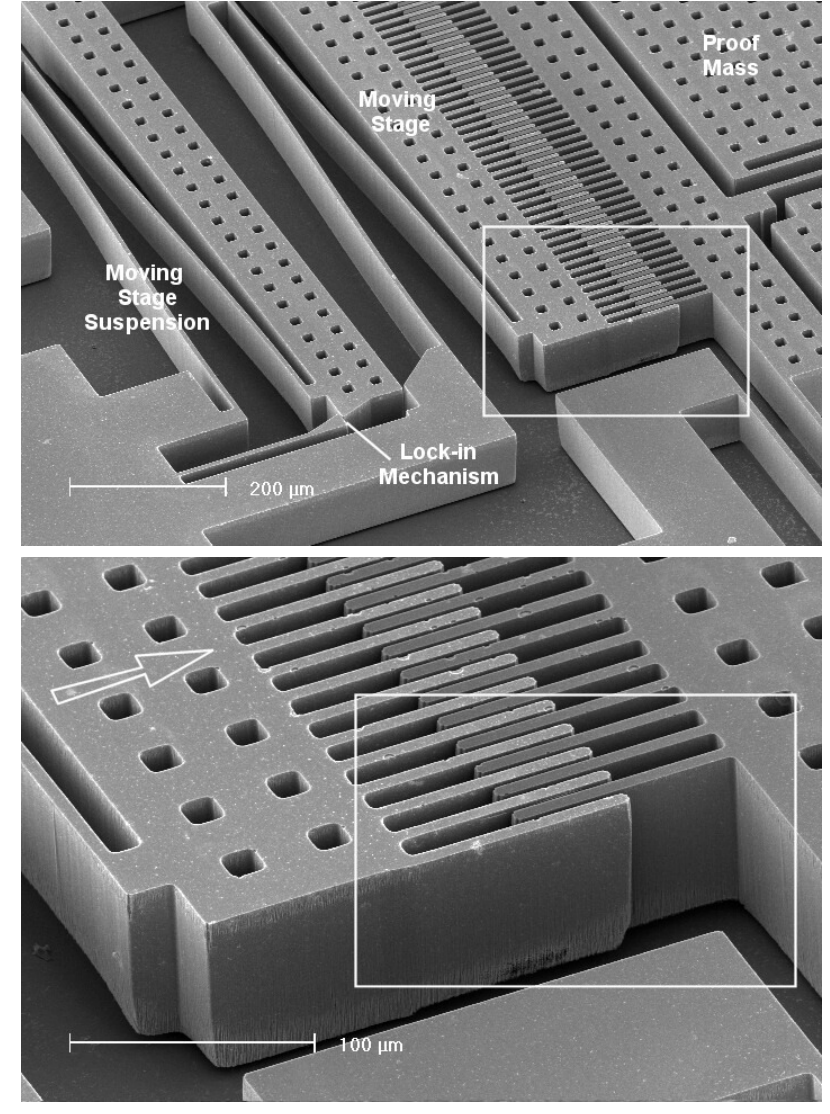

Figure 1. SEM images of post-release positioned comb-drives integrated in a micromachined gyroscope. The resulting finger gap after the assembly is $1 \mu \mathrm{m}$, while the process minimum gap requirement is $10 \mu \mathrm{m}$.

tional and isotropic silicon dry etch and are protected on the sides by refilled trenches. This process involves one layer of low-pressure chemical vapor deposited (LPCVD) silicon nitride, one layer of LPCVD silicon dioxide, and one layer of LPCVD polysilicon [2]. However, this process and similar approaches require additional expensive fabrication steps, and increase the resulting cost.

The post-release assembly technique presented in this paper aims to increase the sensing and actuation capacitances in micromachined devices, without any modification in the fabrication process. With a simple additional assembly step, the performance and noise characteristics are enhanced beyond the fabrication process limitations. 


\section{Enhancement of Comb-Drive Capacitance}

Interdigitated comb-drives are one of the most common actuation structures used in MEMS devices. The primary advantages of comb-drives are long-stroke actuation capability, the linearity of the generated forces, and the ability of applying displacement-independent forces for high-stability actuators. Linearized drive forces along the $\mathrm{x}$-axis can be achieved by appropriate selection of voltages applied to the opposing comb-drive sets. A balanced interdigitated comb-drive scheme is imposed by applying $V_{1}=V_{D C}+\nu_{A C}$ to one set of comb drives, and $V_{2}=V_{D C}-\nu_{A C}$ to the other set, where $V_{D C}$ is a constant bias voltage, and $\nu_{A C}$ is a time-varying voltage. Assuming negligible deflections along the $y$-axis, the net electrostatic force reduces to [4]

$$
F_{c o m b}=4 \frac{\varepsilon_{0} z_{0} N}{y_{0}} V_{D C} \nu_{A C}
$$

where $z_{0}$ is the finger thickness, and $y_{0}$ is the finger separation. It should be noticed that, the net force along the $\mathrm{x}$-axis is independent of the displacement along the $\mathrm{x}$-axis, and the overlap length.

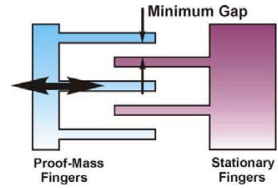

Conventional Comb-drive

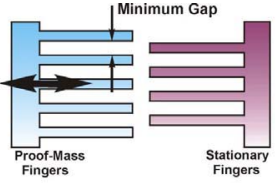

Before Assembly

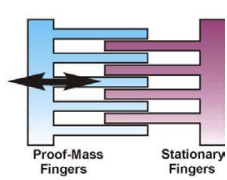

Figure 2. Comparison of a conventional comb-drive structure, and the post-release positioning approach (before and after assembly) designed for the same fabrication process. Notice the difference in the resulting gap, and the number of fingers per unit area.

In conventional interdigitated comb-drives, the gap between each stationary and moving finger is determined by the minimum-gap requirement of the fabrication process (Figure 2). For example, if the minimum gap is $10 \mu \mathrm{m}$, the gap between the conventional comb-drive fingers is $10 \mu \mathrm{m}$.

In the presented post-release positioning approach, the fingers attached to opposing electrodes are designed initially apart, and interdigitated after the release. Thus, the gap between the fingers after interdigitating can be much smaller than the minimum-gap requirement (Figure 2). For the same example, if the width of one finger is $8 \mu \mathrm{m}$ and the minimum gap is $10 \mu m$; the resulting gap between the stationary and moving fingers after the assembly is $1 \mu \mathrm{m}$. This results in 10 times increase in the force per finger. Furthermore, the number of fingers per unit area is increased by allowing smaller gaps. In this example, exactly 2 times as many fingers can be used in the same area, resulting in a total of 20 times increase in the drive-force.

Figures 1 and 3 present the microscope photographs of the assembled post-release positioning comb-drives integrated in a micromachined gyroscope. It is observed that excellent
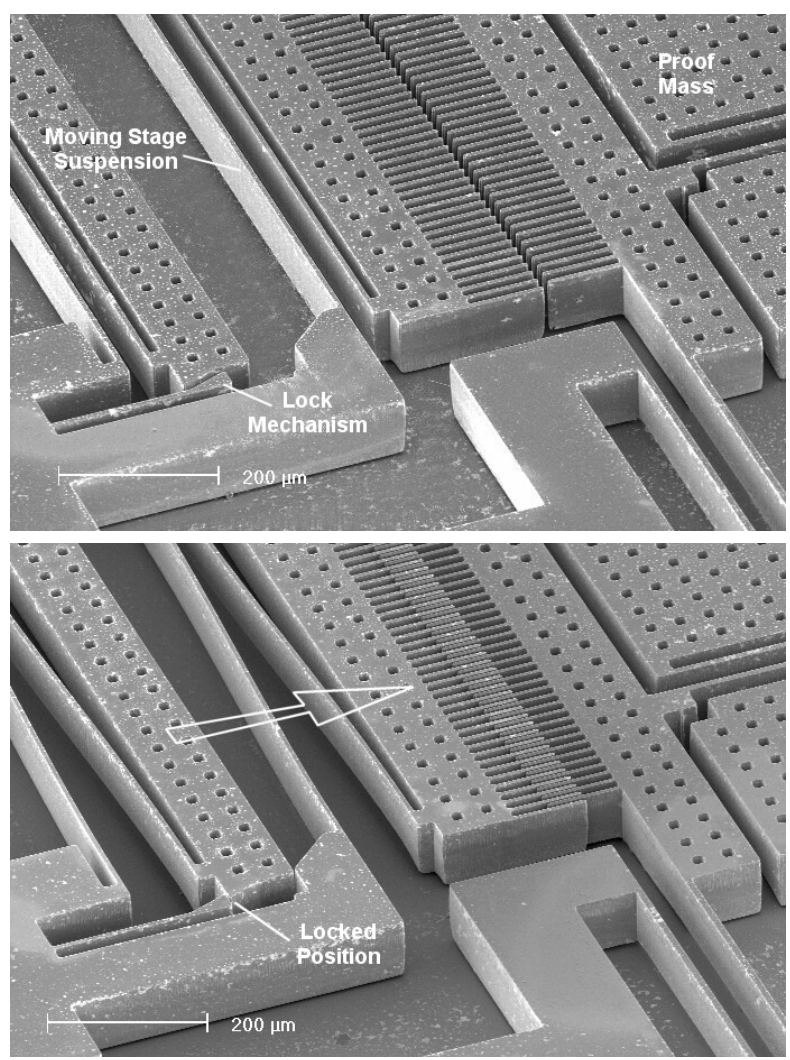

Figure 3. The scanning-electron-microscope images of postrelease positioning comb-drives integrated in a micromachined gyroscope, before and after assembly.

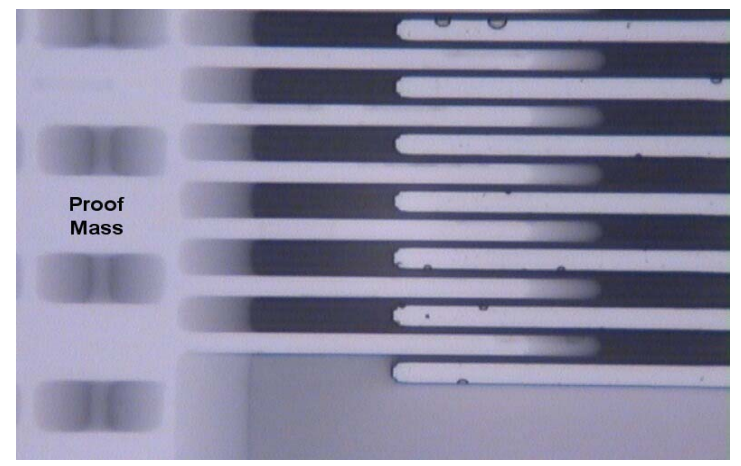

Figure 4. Microscope photographs of the assembled postrelease positioning comb-drives integrated in a micromachined gyroscope. The gyroscope is successfully driven into resonance in the drive-mode with the assembled comb fingers.
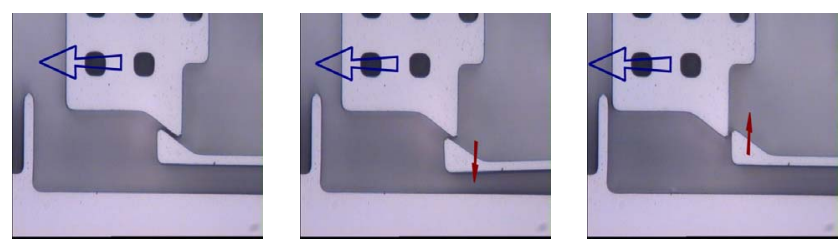

Figure 5. Microscope photographs of the ratchet structure utilized as the locking mechanism for post-release positioning. 
positioning is achieved, providing uniform gaps across the comb-drive structure. Also, the gyroscope proof-mass is successfully driven into resonance in the drive-mode with the assembled comb fingers (Figure 4).
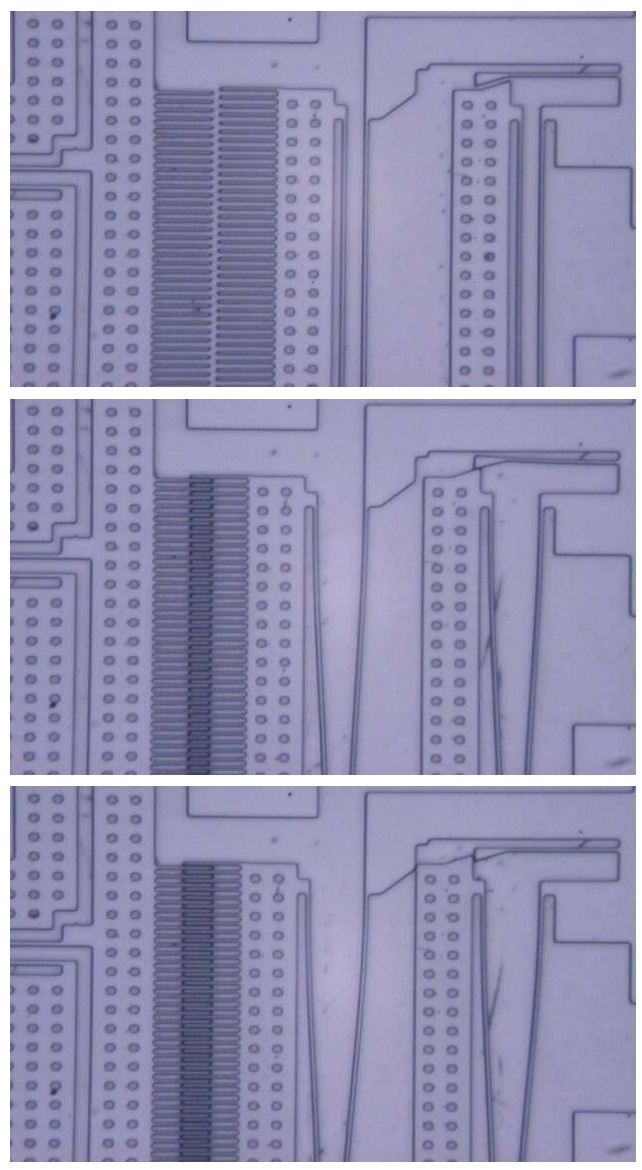

Figure 6. The assembly sequence of the post-release positioning comb-drives using thermal actuators.

Thermal actuators have been successfully used in the fabricated prototypes (Figure 6) for displacing the moving stages and assembling the devices, and other actuation means are possible. The explored locking mechanisms include ratchet structures (Figure 5), and bistable mechanisms (Figure 7). In the prototype gyroscopes, the gyroscope proof-masses were successfully driven into resonance in the drive-mode with the assembled comb fingers, verifying that both the ratchet and bistable lock-in mechanisms provide the required alignment and uniform finger spacing.

\section{Enhancement of Parallel-Plate Capacitance}

Parallel-plate actuation provides much larger forces per area compared to comb-drives, with the expense of limited stable actuation range, and non-linear actuation forces. The net force generated by a parallel-plate actuator is

$$
\overrightarrow{F_{p p}}=-\frac{\varepsilon_{0} V_{D C}^{2} z_{0} x_{0}}{2\left(d_{0}-x\right)^{2}} \hat{e}_{y} .
$$

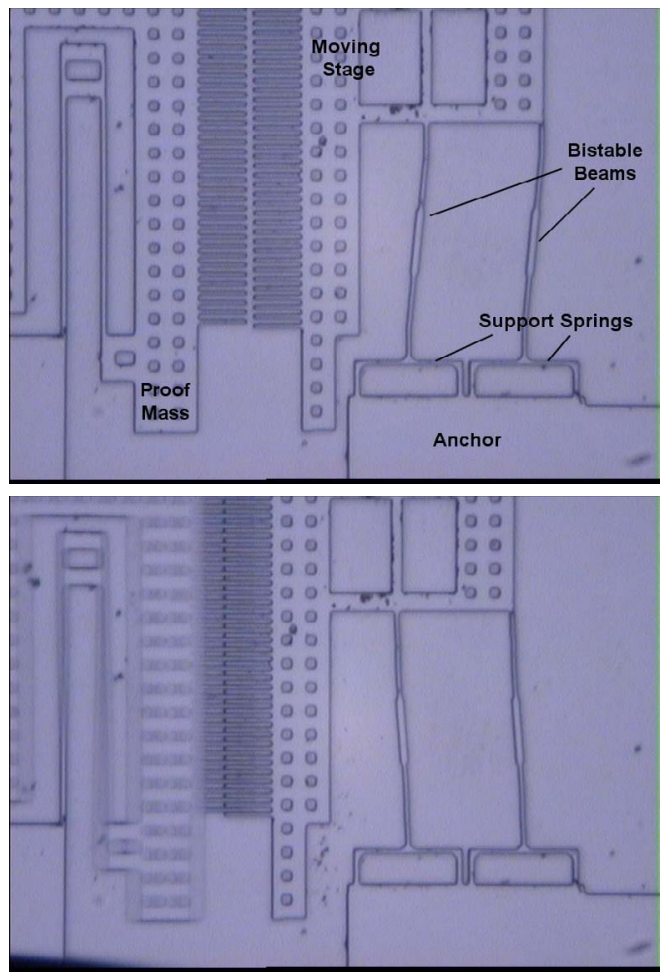

Figure 7. The assembly sequence of the post-release positioning comb-drives with the bistable lock-in mechanism, using thermal actuators. The gyroscope is successfully driven into resonance in the drive-mode with the assembled comb fingers.

where $\varepsilon_{0}=8.854 \times 10^{-12} \mathrm{~F} / \mathrm{m}$ is the dielectric constant, $A$ is the total actuation area, $d_{0}$ is the electrode gap, and $V_{D C}$ is the DC bias voltage. The non-linear electrostatic force profile in parallel-plate actuation electrodes is usually exploited for resonance frequency tuning. The negative electrostatic spring constant that reduces the resonant frequency with increasing DC bias can be found by taking the derivative of the electrostatic force $F_{p p}$ with respect to displacement

$$
k_{e l}=\frac{\partial F_{p p}}{\partial x}=-\frac{\varepsilon_{0} A}{d_{0}^{3}} V_{D C}^{2}
$$

Thus, the shrinking the electrode gap enhances both the actuation force, and the electrostatic frequency tuning range.

\section{Capacitive Detection}

In micromachined inertial sensors, differential capacitance sensing is generally employed to linearize the capacitance change with deflection. Defining $y_{0}$ as the finger separation, $l$ as the length of the fingers, and $t$ as the thickness of the fingers; the differential capacitance values become

$$
C_{s \pm}=N \frac{\varepsilon_{0} t l}{y_{0} \mp y}, \quad \Delta C=C_{s+}-C_{s-}=2 N \frac{\varepsilon_{0} t l}{y_{0}^{2}} y
$$

It is observed that the capacitance change is inversely proportional to the square of the initial gap. Thus, the performance of the sensor (i.e. sensitivity, resolution, and signal to noise 
ratio) is improved quadratically by decreasing the initial gap of the sensing electrodes.

Similarly, the post-release positioning concept is implemented on parallel-plate detection electrodes by attaching the stationary fingers of the sensing electrodes to a moving stage that locks into the desired position before operation. Figure 8 presents the scanning-electron-microscope images of the post-release positioning sensing electrodes integrated in a micromachined gyroscope, before and after assembly. It is observed that excellent positioning is achieved, providing uniform gaps across the sensing electrode structure. Electrode gaps in the order of 1-2 $\mu m$ have been achieved with $100 \mu m$ thick structures (Figure 9), while the minimum-gap requirement of the fabrication process is $10 \mu \mathrm{m}$.

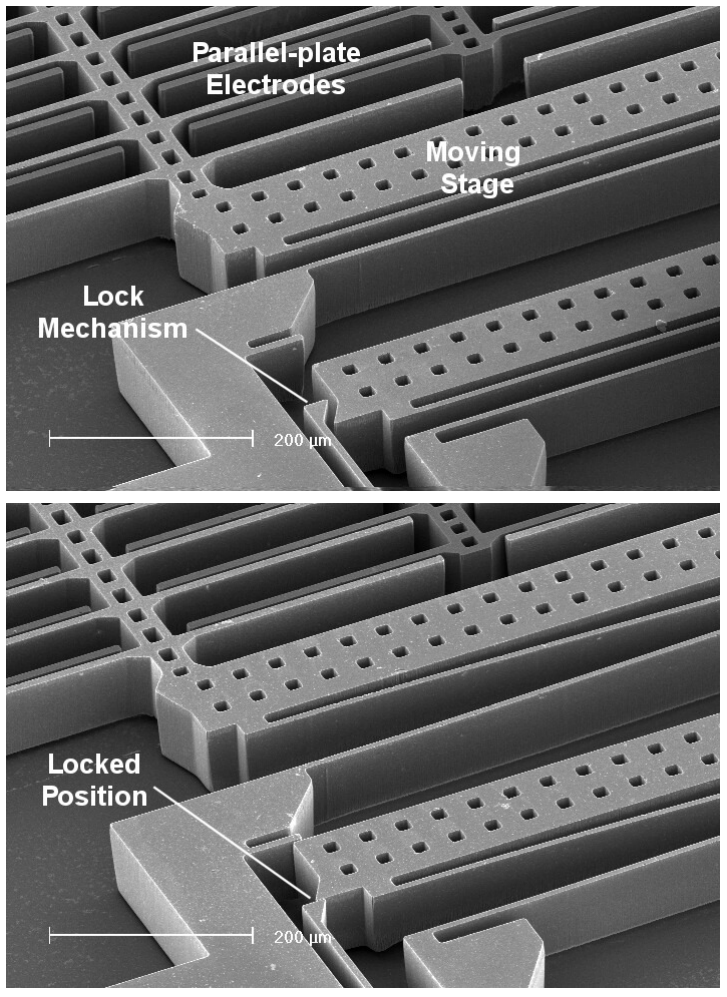

Figure 8. The scanning-electron-microscope images of postrelease positioning sensing electrodes integrated in a micromachined gyroscope, before and after assembly.

\section{IMPLEMENTATION IN A MEMS GYROSCOPE}

The post-release capacitance enhancement technique has been successfully implemented on the comb-drives and the sensing electrodes of bulk-micromachined prototype gyroscopes. System identification algorithms applied on the electrostatically acquired drive-mode frequency responses of the gyroscope revealed that 7.22 times larger actuation force is achieved compared to conventional comb-drives implemented on the exact same gyroscope structure. With the assembled comb-drives, $12 \mu \mathrm{m}$ drive amplitude was achieved with 10V DC and $1 \mathrm{~V}$ AC actuation voltages in air. The gyro-
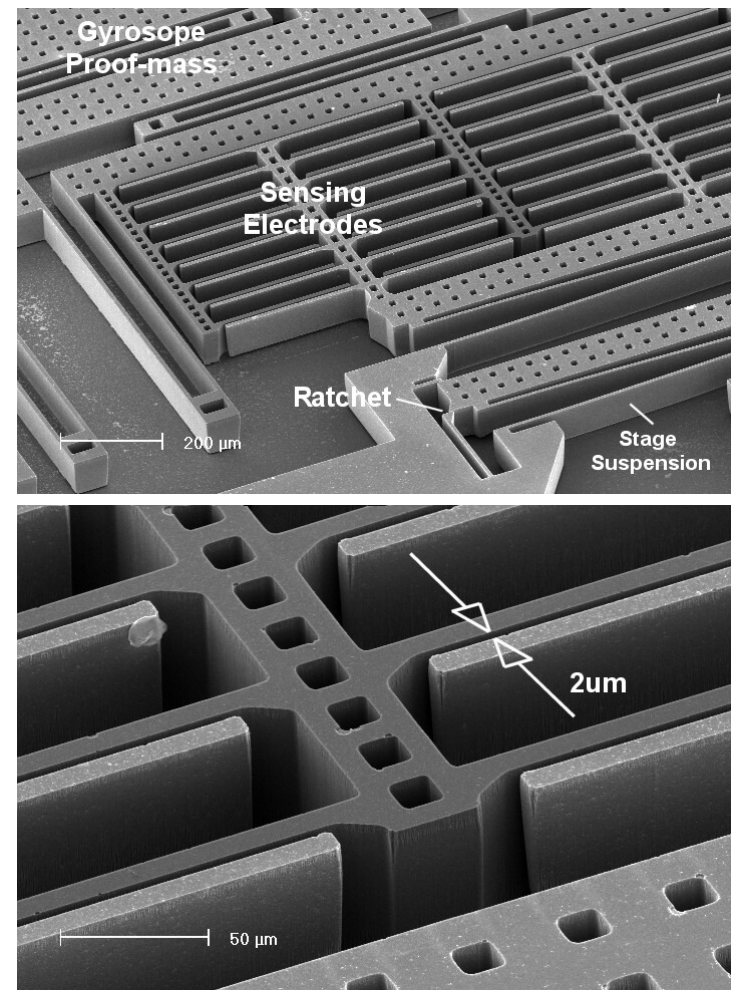

Figure 9. Electrode gaps in the order of $1-2 \mu m$ have been achieved with $100 \mu m$ thick structures, while the minimum-gap requirement of the fabrication process is $10 \mu \mathrm{m}$.

scope with assembled comb-drives and detection electrodes exhibited a sensitivity of $0.91 \mathrm{mV} / 0 / \mathrm{s}$, excellent linearity, and a noise floor of $0.25^{\circ} / \mathrm{s} / \sqrt{\mathrm{Hz}}$ at $50 \mathrm{~Hz}$ bandwidth in atmospheric pressure.

\section{ACKNOWLEDGEMENTS}

This work is supported in part by the National Science Foundation Grant CMS-0409923, program managers Dr. ShinChi Liu and Dr. Masayoshi Tomizuka.

\section{REFERENCES}

[1] T. Hirano, T. Furuhata, K.J. Gabriel, and H. Fujita. Design, Fabrication and Operation of Submicron Gap Comb-Drive Microactuators. Journal of Microelectromechanical Sytems, Vol. 1, 1992.

[2] F. Ayazi, and K. Najafi. High Aspect-Ratio Combined Poly and Single-Crystal Silicon (HARPSS) MEMS Technology Journal of Microelectromechanical Sytems, Vol. 9, 2000, pp. 288-294.

[3] S. Pourkamali, F. Ayazi. SOI-Based HF and VHF Single-Crystal Silicon Resonators With Sub-100 Nanometer Vertical Capacitive Gaps. Proceedings of Solid-State Sensor and Actuator Workshop, 2003.

[4] W.A. Clark, R.T. Howe, and R. Horowitz. Surface Micromachined Z-Axis Vibratory Rate Gyroscope. Solid-State Sensor and Actuator Workshop, 1994. 